\title{
Review of current intracranial aneurysm flow diversion technology and clinical use
}

\author{
Sudeepta Dandapat (1) , ${ }^{1}$ Alan Mendez-Ruiz, ${ }^{1}$ Mario Martínez-Galdámez 다, ${ }^{2}$ \\ Juan Macho, ${ }^{3,4}$ Shahram Derakhshani, ${ }^{3}$ Gustavo Foa Torres, ${ }^{5}$ Vitor M Pereira, ${ }^{6,7}$ \\ Anil Arat, $^{8}$ Ajay K Wakhloo, ${ }^{9}$ Santiago Ortega-Gutierrez (D) 1,10
}

- Additional material is published online only. To view please visit the journal online (http://dx.doi.org/10.1136/ neurintsurg-2020-015877).

For numbered affiliations see end of article.

Correspondence to Dr Santiago Ortega-Gutierrez, Department of Neurology, The University of lowa Hospitals and Clinics, lowa City, IA 52242. USA; santy-ortega@uiowa.edu

SD and AM-R contributed equally.

Received 9 July 2020

Revised 4 September 2020

Accepted 10 September 2020

Published Online First

25 September 2020
Check for updates

(c) Author(s) (or their employer(s)) 2021. No commercial re-use. See rights and permissions. Published by BMJ.

To cite: Dandapat $S$, Mendez-Ruiz A, Martínez-

Galdámez $\mathrm{M}$, et al.

$J$ Neurolntervent Surg

2021:13:54-62.

\section{ABSTRACT}

Endovascular treatment of intracranial aneurysms (IAs) has evolved considerably over the past decades. The technological advances have been driven by the experience that coils fail to completely exclude all IAs from the blood circulation, the need to treat the diseased parent vessel segment leading to the aneurysm formation, and expansion of endovascular therapy to treat more complex IAs. Stents were initially developed to support the placement of coils inside wide neck aneurysms. However, early work on stent-like tubular braided structure led to a more sophisticated construct that then later was coined as a flow diverter (FD) and found its way into clinical application. Although FDs were initially used to treat wide-neck large and giant internal carotid artery aneurysms only amenable to surgical trap with or without a bypass or endovascular vessel sacrifice, its use in other types of IAs and cerebrovascular pathology promptly followed. Lately, we have witnessed an explosion in the application of FDs and subsequently their modifications leading to their ubiquitous use in endovascular therapy. In this review we aim to compile the available FD technology, evaluate the devices' peculiarities from the authors' perspective, and analyze the current literature to support initial and expanded indications, recognizing that this may be outdated soon.

\section{INTRODUCTION}

With the introduction of detachable coils by Guido Guglielmi in the early $1990 \mathrm{~s}^{1}$ and the addition of adjunctive devices (intracranial stents, balloons, and pre-shaped and shapeable microcatheters), endovascular therapy became an alternative to surgery for ruptured and unruptured intracranial aneurysms (IAs) that required treatment. ${ }^{23}$ However, a complete aneurysm obliteration rate was achieved in one third of the patients, with major recurrences seen in one-fifth of all treated cases. ${ }^{4}$ These rates might be magnified in the context of larger wideneck aneurysms due to their complex aneurysm morphology and neck geometry, as well as the existence of concomitant disease of the parent vessel.

Coiling failures are related to coil compaction inside the aneurysm pouch, due to secondary material fatigue associated with forces generated on the coil mass with each pulse cycle, resulting in aneurysm recanalization and in some cases aneurysm regrowth. ${ }^{5}$ With the necessity to expand therapeutic alternatives, the hypothesis of vessel reconstruction with the use of endoluminal implants led to the development of flow diverter devices. ${ }^{67}$ Encouraged by these early amorphous but compelling findings, Wakhloo and Lieber drove the development of endoluminal scaffolds for vascular reconstruction and coined them as "flow diverters" (FDs). ${ }^{8}$

In this review, we highlight the essential technical features of currently available FDs, review essential clinical literature supporting their use, and review the published data for important off-label indications.

\section{METHODS}

For this study we conducted a literature search of the electronic databases PubMed, EMBASE and Google Scholar on FD devices. The key words and free text search used in combination (using the Boolean operators "or" and "and") were the following: "flow diverter", "flow diversion", "intracranial aneurysm(s)", "cerebral aneurysm(s)", "carotid fistula(s)", "posterior circulation", "Pipeline", "Surpass", "FRED", "Silk", "Tubridge", "p64", and "p48". The inclusion criteria for the studies were the following: (a) meta-analysis, prospective and retrospective studies; (b) case series of at least five patients undergoing intracranial aneurysm treatment with an FD; (c) duration of study follow-up of at least 1 month; (d) angiographic follow-up rates of aneurysm occlusion; (e) documenting follow-up complications and mortality rates. The exclusion criteria were studies published in languages other than English or without an English translation.

\section{CONCEPTS OF ENDOLUMINAL FLOW DIVERSION Device properties}

The primary intention of FD is to optimally alter the flow between the parent vessel and the aneurysm while providing an endovascular scaffold for the vessel to heal the defect that is responsible for the aneurysm to form in the first place. The degree by which these objectives are met are based on a complex interaction between the FD properties, parent vessel anatomy, aneurysm size, side branches and perforators. Early in vivo and in vitro studies showed that the most relevant FD properties during this process are porosity and pore density. ${ }^{9} 10$ Porosity is defined as the ratio of the metal-free surface area to the total surface area of the device, whereas pore density is the number of pores per unit surface area. 


$$
\text { porosity }(\%)=\frac{\text { Total surface area }- \text { Metal surface area }}{\text { Total surface area }} \times 100
$$

Bench top studies using laser induced fluorescence (LIF) imaging and particle image velocimetry (PIV), computational fluid dynamics (CFD) as well as experiments in the rabbit elastase aneurysm model demonstrated that a maximum of $70 \%$ porosity and a minimum pore density of 18 pores $/ \mathrm{mm}^{2}$ were ideal parameters to achieve a high rate of stable aneurysm occlusion and yet preserve side branches covered by FDs. ${ }^{911}{ }^{12}$ Other combinations of porosity and pore density achieved similar stable aneurysm occlusion at longer time periods of 3 to 6 months. ${ }^{9} 10$ Translating these properties to in vivo models, while interacting with the underlying parent vessel anatomy aneurysm and vessels arising from the aneurysm neck, represents a key feature for an FD to be successful in clinical practice.

Another factor to consider for the success of an FD is the predictability of the device behavior on deployment. The intravascular change in the length of an FD, known as foreshortening, could represent an issue in the clinical practice if the change is not as expected, leading to an under- or overestimation of the size of the FD. ${ }^{1314}$ However, with the introduction of virtual simulation tools, operators now have the advantage of anticipating the FD behavior prior to the procedure. These instruments could help bridge the gap between the FD manufacturer-recommended size and a real-world scenario. ${ }^{13} 14$

\section{Mechanism of action}

The mechanism of action of FD can be divided into three stages: hemodynamic, thrombus formation, and endothelialization. ${ }^{15}$ The hemodynamic stage happens immediately after FD placement, which exerts a disruption of blood flow into and out of the aneurysm from the parent artery related to the resistance (impedance) created by the mesh. Even though contrast opacification and/or washout may be visualized on angiograms, a marked reduction in velocity of blood flow and shear stresses occurs inside the aneurysm (figure 1I). ${ }^{12}$ This is followed by immediate activation of platelets via a complex pathway with progressive formation of a stable thrombus thrombus formation stage) over days to weeks. The thrombosis and subsequent occlusion of the aneurysm depends on the (neck) size of the aneurysm, FD properties, the subject's blood rheology, and the platelet response to antiplatelet medication. During this stage there may be worsening of local mass effect and/or inflammation leading to exacerbation of prior symptoms such as headaches if previously present. The endothelialization stage represents the transformation of the amorphous thrombus to its final collagen stage and the simultaneous and progressive endothelialization of the FD driven by CD34+ endothelial progenitor cells that can take several months to years. ${ }^{16}$ The FD acts as a scaffold for neo-endothelization and remodeling of the artery. Time sensitive intra-aneurysmal thrombus transformation to collagen leads to a final reduction in aneurysmal mass. ${ }^{9} 17$

\section{Platelet inhibition}

Given the metallic properties of the FD, dual antiplatelet therapy (DAPT) is required to reduce the risk of thromboembolism. Aspirin and clopidogrel are the most commonly used antiplatelets; however, due to the rising variability in clopidogrel platelet inhibition, platelet function testing (PFT) has been
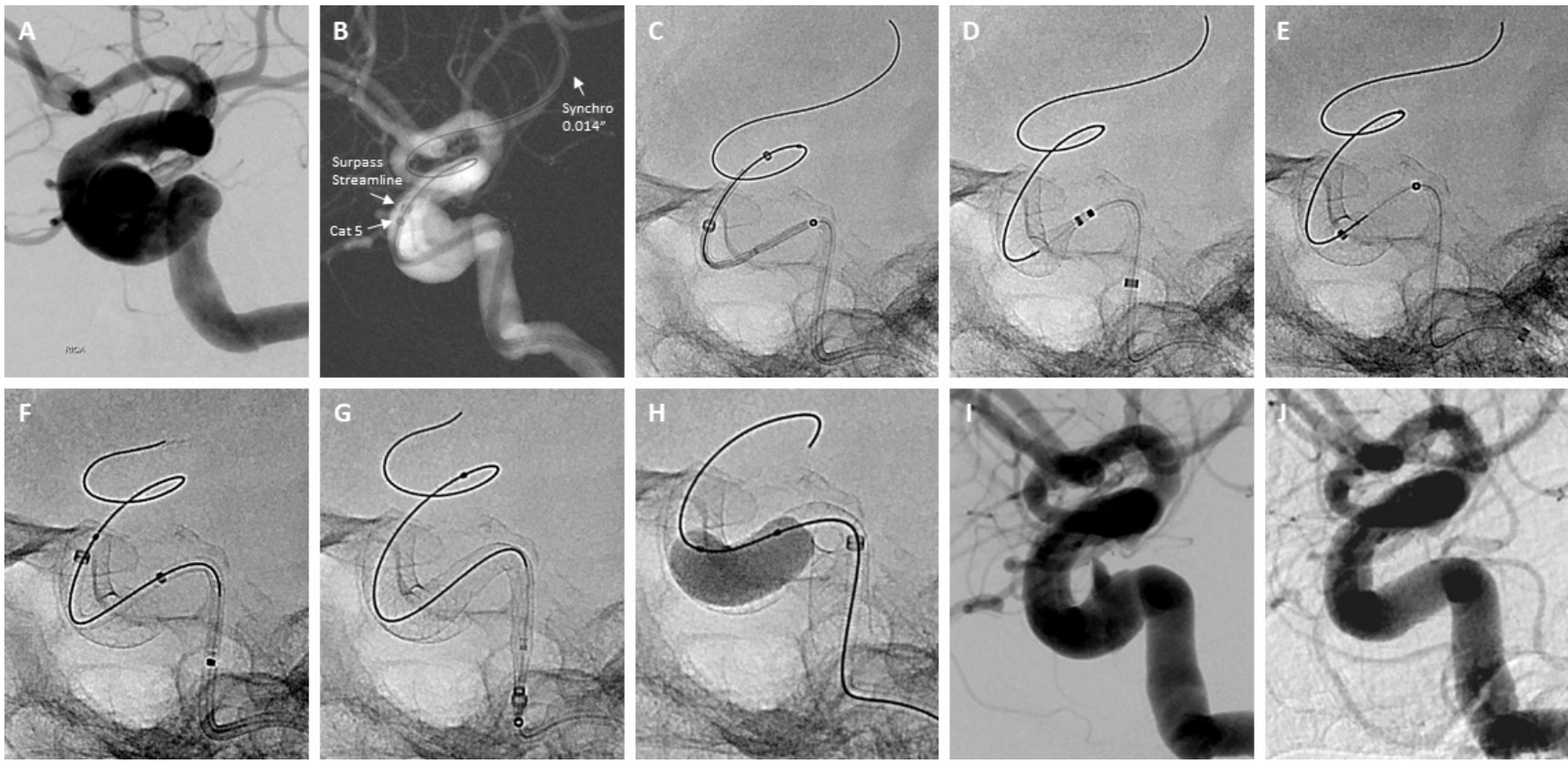

Figure 1 Embolization of right ICA cavernous segment aneurysm with Surpass Streamline OTW system. (A) Preoperative angiogram with right ICA cavernous aneurysm. (B) Placement of Cat 5 intermediate catheter distal to the aneurysms and advancement of the Streamline catheter containing the Surpass FD over Synchro microwire 0.014 inch. (C) Position of the Streamline catheter containing Surpass FD into the supraclinoid segment. (D) Unsheathing of FD at the distal target site first within Cat 5 (not shown) and then pushing the Surpass FD to allow further expansion for a defined proximal landing zone vessel wall apposition. (E) Maintaining of wire position and re-accessing the FD with the delivery Streamline catheter. (F) Selection of second FD for telescoping placement, and maintaining wire position and FD access with Cat 5. (G) Second FD advanced through the Cat 5 and over the microwire and deployed in a similar fashion. (H) Balloon angioplasty for FD wall apposition optimization. (I) Final angiography with partial contrast filling of aneurysm. (J) Complete obliteration of cavernous aneurysm at 6 month follow-up angiogram. FD, flow diverter; ICA, internal carotid artery; OTW, over-the-wire. 
increasingly used to ensure therapeutic inhibition and mitigate the risk of thrombotic/hemorrhagic complications. Nevertheless, PFT has been the subject of controversy, with association of harmful outcomes in FD patients as observed in a meta-analysis by Brinjiki et al. ${ }^{18}$ Yet, the utility of PFT to prognosticate complications may have become more meaningful in recent years, as observed in a meta-analysis by Ajadi et al in which platelet hyporesponders and hyper-responders were associated with thrombotic and hemorrhagic events, respectively, following FD. ${ }^{19}$ The risk of thrombotic complications in platelet hypo-responders has also been shown to be lower when antiplatelet medication is modified. ${ }^{20}$ At times, more predictable oral antiplatelet medication such as ticagrelor and prasugrel are added to substitute for clopidogrel in platelet hypo-responders or even used directly as first line antithrombotic agents. Lastly, PFT has been associated with better functional outcomes after neurovascular stenting of IA. ${ }^{21}$ Prospective studies are needed to fully assess the clinical benefit of PFT.

\section{EVOLUTION OF DEVICES}

Based on the previous benchwork studies, various companies embarked on the development and manufacturing of FDs for clinical applications. The Pipeline Embolization Device (PED, Chestnut Medical Technologies, Menlo Park, CA) was the first commercially available $\mathrm{FD}$, which received the Conformité Européene (CE) mark in June 2008 and entered the US market after receiving Food and Drug Administration (FDA) approval on April 6, 2011. Since then we have witnessed an exponential growth in technological advancements and applications of several FDs. Aside from their intrinsic features, the design of FDs is primarily based on the use of cobalt chromium (Pipeline and Surpass) or nitinol (all the others). Cobalt/chromium add stiffness and radial force while nitinol brings flexibility and easy navigation and deployment. Cobalt/chromium implants allow a better answer to ballooning when an incomplete wall apposition is observed. Online supplemental table 1 summarizes the main technical characteristics and clinical evidence for the safety and efficacy of each device.

\section{Pipeline}

Pipeline Embolization Device

The PED consists of a braided stent of 48 wires of cobaltchromium-nickel alloy including 12 wires of platinum-tungsten that serve as marker wires. It is deployed with a combination of pushing and unsheathing techniques through a 0.027 inch microcatheter.

Since its introduction, the PED has shown a high rate of stable internal carotid artery (ICA) aneurysm occlusion. The PED for the Intracranial Treatment of Aneurysms (PITA) trial ${ }^{22}$ was the first multicenter prospective study done using the PED in centers of Europe and South America in 2011, in which Nelson et al reported a complete aneurysm occlusion of $93.6 \%$ at 6 month follow-up in mostly medium-sized, unruptured ICA aneurysms, with a low morbidity of $6.5 \%$ and $0 \%$ mortality rates. ${ }^{22}$ The Pipeline of Uncoilable or Failed Aneurysms (PUFS) trial was the first multicenter study done in the USA for large and giant $(\geq 10 \mathrm{~mm})$ wide-necked aneurysms of the ICA demonstrating complete occlusion rates of $73.6 \%, 86.8 \%, 93.4 \%$ and $95.2 \%$ at 6 months, 1 year, 3 years and 5 years, respectively. ${ }^{23}$ Based on the PUFS study, PED received FDA approval in 2011 for the treatment of wide-neck large aneurysms of limited segment of the ICA. $^{24}$ Subsequent real-world experience in the International Retrospective Study of the Pipeline Embolization Device
(IntrePED) and in the Aneurysm Study of Pipeline in an Observational Registry (ASPIRe) studies demonstrated that while the Pipeline FD was quite effective with an overall complete obliteration rate up to $74.8 \%$, giant aneurysms were associated with higher rates of morbidity and mortality up to $25.8 \% .^{2526}$

\section{Pipeline Flex Embolization Device}

A second-generation FD, the Pipeline Flex Embolization Device (Pipeline Flex) (Medtronic) was first introduced in Europe, receiving CE approval in 2014, andFDA approval in 2015 in the USA. Although the stent itself did not have any differences with its predecessor, the delivery system featured two major characteristics: $2-3 \mathrm{~cm}$ polytetrafluoroethylene (PTFE) protective sleeves that replaced the capture coil originally found in the PED, and a resheathing pad that allows device recovery and redeployment. These features allowed for a decrease in technical complications and malposition rates as observed in a multicenter US study compared with previous trials using first generation PED. ${ }^{27}$ The PREMIER study evaluated the use of Pipeline Flex in the treatment of small to medium sized $(<12 \mathrm{~mm})$ unruptured wide neck aneurysms in primarily the ICA ( $95 \%$ of the aneurysms) and vertebral artery. At 1 year follow-up, $76.8 \%$ of patients had achieved the study's primary effectiveness endpoint of complete aneurysm occlusion without significant parent artery stenosis or retreatment. The combined major morbidity and mortality rate was $2.1 \% .^{28}$

\section{Pipeline Flex Embolization Device with Shield Technology}

A third device, the Pipeline Flex with Shield Technology (Pipeline Shield) (Medtronic), was developed in an attempt to decrease thromboembolic complications. Receiving CE approval in 2015, the Pipeline Shield has the same design and configuration as the Pipeline Flex but features a surface modification whereby a synthetic layer of phosphorylcholine coats the metal strands with the aim of reducing thrombogenicity. In in vitro studies, the device has been observed to be less thrombogenic than previous generation Pipeline Flex and the Flow Re-Direction Endoluminal Device (FRED). ${ }^{29}$ The constraints that the phosphorylcholine surface modifications might exert on the opening and apposition of the device are currently under evaluation.

Although the concept of the Pipeline Shield to decrease the risk of thromboembolic complications seems promising, the rates of thromboembolic events reported in two large multicenter studies of unruptured IA (6.4\% and 3.3\%) were not lower than those of the PREMIER (2.1\%) and IntrePED (3.3\%) when DAPT was used. ${ }^{30} 31$ Large prospective trials with single antiplatelet therapy (SAPT) are needed to determine the superiority or not of the Pipeline Shield.

Further information on acutely ruptured IA using Pipeline Shield with SAPT is described in the section "FD in acutely ruptured IA".

\section{Surpass}

\section{Surpass Streamline}

The Surpass Streamline (SS) FD is braided out of cobaltchromium alloy with 12 platinum-tungsten alloy marker wires; it was developed by Surpass Medical, Ltd (Tel Aviv, Israel) and acquired by Stryker (Stryker Neurovascular, Fremont, CA) in 2012. SS received the CE mark in 2010 and FDA approval in 2018. The wire braid angle as well as the high number of wires preserves the structural integrity of the device, the diamond cell shape of the implant and mesh density, regardless of large variabilities in the arterial diameter over the length of the FD. ${ }^{32} 33$ 
Consistent mesh density ensures a more even effect on flow reduction, preventing pockets of increased flow impingement zones within the aneurysm. Unlike other products, the device is manufactured in diameters of $1 \mathrm{~mm}$ increments. The number of wires for each device vary depending on the device diameter to maintain biomechanical stability and a consistent reduction of aneurysm inflow (up to 96 wires for the largest device diameter of $5.3 \mathrm{~mm}$ ). The wire diameter used for braiding ranges from 25-32 $\mu \mathrm{m}$ for currently available implants. The mesh density of the device ranges from $21-32$ pores $/ \mathrm{mm}^{2}$.

The foreshortening percentage of a Surpass FD varies, like for all braided implants, and depends on the device diameter and length, with approximately 38\%, 42\% and 26\% foreshortening under nominal diameter experienced for $3 \mathrm{~mm}, 4 \mathrm{~mm}$ and $5 \mathrm{~mm}$ systems, respectively. This foreshortening is found to be less significant due to the braid angle and larger number of wires, which also helps prevent kinking and torquing of the FD and facilitate device opening in tortuous vasculature. The larger number of wires, however, impedes the delivery of the implant through an empty microcatheter as compared with other FDs, and requires a separate delivery system for each device. The implant comes preloaded in the 0.040 inch delivery microcatheter that renders overall stiffness to the system, and thus requires higher manual push force and a tri-axial approach for device deployment. A long sheath and an intermediate catheter are used for navigation of the delivery system beyond the aneurysm neck. A 5 French intermediate catheter is recommended for device deployment of the initial FD portion (figure 1C,D). Furthermore, a 0.014 inch microwire is placed through the center of the delivery system (over-the-wire (OTW)) for added support and navigation. Yet, the presence of the central wire carries the risk of distal vessel perforation. ${ }^{34}$

The combination of OTW and medium braid angle make SS a stable device in the absence of arterial wall situations such as fusiform aneurysms with very wide necks, since it does not significantly foreshorten from its nominal length and telescopes with ease if necessary.

The Surpass Intracranial Aneurysm Embolization System Pivotal Trial to Treat Large or Giant Wide Neck Aneurysms (SCENT) was a large, prospective, multicenter trial for the safety and effectiveness of the SS in the treatment of aneurysms in difficult-to-treat extra- and intradural locations. The results showed a complete occlusion rate of $66.1 \%$ and $8.3 \%$ morbidity and mortality rates, respectively, at 1 year follow-up. ${ }^{34}$ This study served as the basis for FDA approval of SS in the treatment of large and giant wide-neck aneurysms in the ICA up to the terminus.

\section{Surpass Evolve}

A second-generation device, the Surpass Evolve (Stryker) received CE approval in 2019 and FDA approval in May 2020. In comparison to its predecessor, the Surpass Evolve has 48 wires for the $2.5 \mathrm{~mm}$ device and 64 wires for the $3.25-5 \mathrm{~mm}$ implants. The mesh density of Surpass Evolve (15-30 pores/ $\mathrm{mm}^{2}$ ) is comparable to that of SS despite its reduced wire count. This is achieved by a higher braid angle in its design. This feature provides flexibility to the device for a better vessel wall apposition, an issue that was observed at times at the proximal and distal edges of SS. Additionally, instead of being a preloaded system, Evolve can be used through an empty 0.027 inch microcatheter such as the Excelsior XT-27 microcatheter. The strength of the device is based on its greater radial force for opening and stabilization at the distal end and on the pusher system, where the device is not fixed directly to the wire, but within a plastic cylinder. These features allow for some wire torquing releasing tensions in the system when tracking.

The first clinical experience with Surpass Evolve was reported by Orru et al in a preliminary prospective study published in 2020 for the treatment of mostly small ICA aneurysms. The complete occlusion rate was $57 \%$ with a morbidity rate of $4 \%$ at 4 months follow-up. Due to the smaller delivery system and lower wire count, it was found to be easier to navigate while maintaining its twist resistance, distal aneurysm re-access and precision in deployment. ${ }^{35}$ The EVOLVE trial, a prospective, multicenter, single arm open-label study, started patient enrollment in July 2020 with the intention to treat 235 patients and better evaluate the safety and efficacy of this technology.

\section{FRED}

The FRED (Microvention, Aliso Viejo, California) received CE approval in 2012 and FDA approval in December 2019. It is a self-expanding dual-layer braided stent consisting of a low porosity (48 nitinol wires) inner layer and a high porosity (16 nitinol wires) outer layer. The inner and outer layers are connected by an interwoven tantalum layer that provides radiopacity. The dual layer is only present in $80 \%$ of the central portion of the device with the aim of increasing coverage across the aneurysm neck, and easier opening in distal and proximal landing zones. It is delivered through a 0.027 inch microcatheter using a traditional triaxial system.

The European Flow-Redirection Intraluminal Device (EuFRED) and the Safety and Efficacy Analysis of FRED Embolic Device (SAFE) studies showed a complete aneurysm occlusion of $91.3 \%$ and $73.3 \%$ at 1 year follow-up, respectively. Permanent morbidity and mortality rates for both studies were $<3 \%$ and $<2 \%$, respectively. ${ }^{36} 37$

\section{FRED Jr.}

The FRED Jr. (Microvention) is a CE and FDA approved smaller version (diameter $2.5-3 \mathrm{~mm}$ ) of the FRED for the treatment of aneurysms in small vessels $(2-3 \mathrm{~mm})$. The main difference with the FRED is that the inner layer of the device is composed of 36 wires and the outer layer of 16 wires and it is deployed using a 0.021 inch microcatheter.

In a multicenter study using FRED Jr. in small arteries, Möhlenbruch et al showed a complete aneurysm occlusion rate of $70 \%$ at 6 months follow-up. A 7.1\% periprocedural thromboembolic event rate was observed and no mortality was reported. ${ }^{38}$

\section{Silk}

Silk (Balt Extrusion; Montmorency, France) was the first FD approved by the CE in 2008 for the treatment of IA. Silk is a braided flexible mesh stent made of 44 nitinol and four platinum wire strands with flared ends that act as radiopaque markers. The device is characterized by its high flexibility to adapt to the arterial anatomy. However, this could limit its pushability and trackability in tortuous vessels, with poor opening on deployment due to lower radial force. This also leads to less device stability in very wide necks, particularly in large/giant aneurysms. ${ }^{39}$ The device is delivered through a 0.021 inch microcatheter.

Most of the data on Silk are from retrospective studies with one prospective study performed by Lubicz et al in $2010 .{ }^{40}$ Thirty-four aneurysms in 29 patients were attempted to be treated with a $90 \%$ technical success rate. The complete occlusion rate was $69 \%$ with mortality and morbidity rates of $4 \%$ and $15 \%$, respectively. In the recent largest retrospective study by Foa Torres et al, 246 consecutive patients were treated with the 
Silk device, achieving a complete occlusion rate of $93.9 \%$ with morbidity and mortality rates of $4.2 \%$ and $2.1 \%$, respectively, at 1 year follow-up. ${ }^{41}$

\section{Silk+}

The Silk+ (Balt Extrusion) is a second-generation device, with more enhanced sliding capacity and radiopacity than its predecessor, that received CE approval in 2012. It is made of 48 wires with eight platinum wires and four platinum coils to promote its visibility and flared ends to increase wall apposition. Silk+ can be delivered by 0.021 and 0.025 inch microcatheters with availability in tapered sizes. The need for tapered devices arose due to the often encountered significant difference in the sizes of distal and proximal parent artery, frequently needing placement of two FD to provide adequate coverage.

In 2015, Lubicz et al reported a single center experience with the use of both the Silk and Silk + in 58 patients with 70 aneurysms. At a mean follow-up of 22 months, the complete aneurysm occlusion rate was $73 \%$. The overall permanent neurologic morbidity rate was $5.5 \%$ and there was no procedure-related mortality. All clinical complications (15\%) were seen with the first-generation Silk FD. ${ }^{42}$

\section{Silk Vista Baby}

The Silk Vista Baby (SVB) FD (Balt Extrusion) received CE approval in 2018. It is a low profile (diameter $2-3 \mathrm{~mm}$ ) newer generation device designed to target small distal vessels (1.5$3.5 \mathrm{~mm}$ ). The device is delivered through a 0.017 inch microcatheter. It features a drawn filled tubing (DFT) technology of 48 nitinol wires filled with platinum to enhance radiopacity. A larger diameter new version called Silk Vista with diameters of 3.5 to $4.75 \mathrm{~mm}$ has just received CE mark approval.

Martinez-Galdamez et al reported the periprocedural outcomes of SVB in a series of 41 patients with 43 small aneurysms (mean $9.5 \mathrm{~mm}$ ) at and beyond to the Circle of Willis. The mean parent artery diameter was $2.28 \mathrm{~mm}$ proximally and $2 \mathrm{~mm}$ distally. The intraoperative complete occlusion rate was $18.6 \%$. There were five cases of intraprocedural complications with no clinical consequence. ${ }^{43}$ Schob et al reported the short-term outcomes of 25 patients treated with SVB for aneurysms beyond the Circle of Willis. The complete occlusion rate was $68 \%$ at a mean follow-up of 2.7 months. No procedure-related complications were reported. ${ }^{44}$

\section{p64}

The p64 FD (Phenox, Bochum, Germany) was approved by the CE in 2012. The device consists of a braided mesh stent composed of 64 nitinol wires and is available in $2.5-5 \mathrm{~mm}$ diameters. It is delivered through a 0.027 inch microcatheter and features a mechanical detachment system which allows for device recovery after full deployment.

The p64 has shown high occlusion rates $>85 \%$ at $\geq 12$ months follow-up in unruptured anterior IA, yet the mid-term occlusion rates are still inconclusive with rates of $66.6 \%$ and $82 \%$ at 6 months follow-up in two multicenter studies. Overall, both studies reported a low morbidity $(<3 \%)$ and $0 \%$ mortality rates. $^{45} 46$

\section{p48MW}

The p48MW FD (Phenox) is a CE marked smaller profile device (diameter $2-3 \mathrm{~mm}$ ) designed for the treatment of small distal vessels $(1.75-3 \mathrm{~mm})$. It is a self-expanding nitinol/platinum stent composed of 48 wires with DFT technology. There is an inner distal movable wire (MW) with an atraumatic nitinol tip to prevent rupture of small distal vessels. The device is compatible with a 0.021 inch microcatheter. An alternate version is available without the central MW (p48), compatible with a 0.017 inch microcatheter. Unlike the p64, the p48MW is not mechanically detached.

Bhogal et al reported the safety and efficacy of the device in 25 patients with 25 aneurysms in small vessels. The median parent vessel diameter was $2.4 \mathrm{~mm}$ (range $1.4-3.6 \mathrm{~mm}$ ) proximally and $2.1 \mathrm{~mm}$ (range $1.5-3.4 \mathrm{~mm}$ ) distally. A $70 \%$ complete aneurysm occlusion rate was observed at a mean follow-up of 13.1 months. One patient developed an ischemic event with full recovery at 90 days and one patient died of a cause not related to the treatment. ${ }^{47}$

\section{p48_HPC}

With the same configuration as the p48MW, the p48_HPC (Phenox) is an enhanced device coated with a glycan-based Phenox patented hydrophilic polymer coating (HPC) to reduce thrombogenicity. ${ }^{48}$ To evaluate the safety and efficacy of the device with SAPT in unruptured aneurysms, Bhogal et al reported the initial human experience in a small series of five patients with five aneurysms. The majority were small saccular aneurysms of the anterior circulation. All patients were prescribed prasugrel. At 8.5 months follow-up three aneurysms were completely occluded. No thromboembolic events were reported and one patient developed a right Sylvian fissure hematoma. No mortality was reported. ${ }^{49}$ P48_HPC in ruptured IA is explained in more detail in the "FD for acutely ruptured IA" section.

\section{Derivo}

The first generation of Derivo FD (Acandis GmbH, Pforzheim, Germany) was approved by the CE in 2012. A second generation Derivo (Acandis $\mathrm{GmbH}$ ) was developed to improve device flexibility and visibility in 2017. It consists of 48 nitinol wires with an inner platinum core and three radiopaque markers at each end for increased radiopacity. Additionally, a blue-colored layer of oxides and oxynitrates (BlueXide) coats the device to increase trackability and reduce thrombogenicity. ${ }^{50}$

To date, only one large prospective trial (BRAIDED) for the Derivo has been published showing good mid-term (6 months) and long-term (1 year) occlusion rates $(80.7 \%$ and $89.2 \%$, respectively). This trial was done for mainly unruptured aneurysms with DAPT. The thromboembolic event rate was $2.6 \%$ and a mortality rate of $1.4 \%$ mortality was reported. ${ }^{51}$ The Derivo in acutely ruptured IA is discussed below.

\section{Tubridge}

The Tubridge (MicroPort, Shanghai, China) is a braided, selfexpanding device approved by the National Medical Products Administration (NMPA) in China in 2018. The device consists of 46 braided nitinol wires and two platinum strands for smaller versions $(2.5-3 \mathrm{~mm})$, whereas 62 nitinol wires and two platinum strands constitute larger versions $(3.5-6.5 \mathrm{~mm})$. It is delivered through a 0.029 inch microcatheter. The device holds a radiopaque marker to indicate the resheathing point limit. The safety and efficacy of the device are limited to the scarce clinical evidence available.

In a single center experience using the Tubridge, Zhou et al reported a $72 \%$ complete occlusion rate in 28 patients with 28 large/giant ICA aneurysms at a mean follow-up of 9.9 months. No procedure-related morbidity or mortality were reported. ${ }^{52}$ 


\section{EXPANDED OFF-LABEL INDICATIONS}

A better understanding of FD hemodynamic modification properties and an increased technical expertise have opened a window of opportunity to implement the FD technology to treat pathologies where current endovascular and open surgical options were limited.

\section{Flow diverters at and beyond to the circle of Willis}

FD treatment of distal IA can be technically difficult due to the unfavorable configuration of the vascular anatomy. The small size of parent vessels (diameter $\leq 3 \mathrm{~mm}$ ) and the presence of important divisions and branches represent a risk for ischemic complications when using FD. ${ }^{385354}$ Although promising results have been observed with the use of FD in distal aneurysms with occlusion rates up to $82 \%,{ }^{55}$ clinical and technical complications still exist. In a single center experience, $8.3 \%$ of patients developed postoperative ischemic stroke following PED placement in the A1 segment. ${ }^{53}$ In another study where PED was used in more distal segments A2, M2 and P2, a 7.7\% ischemic and hemorrhagic event rate was observed, of which $4.6 \%$ accounted for aneurysms located at M2 segments. ${ }^{54}$ In fact, the middle cerebral artery has been associated with a higher risk of thromboembolic complications. ${ }^{3855}$
Another important anatomical consideration is that the tortuous anatomy of distal vessels markedly foreshortens the FD on deployment. ${ }^{44}$ This, coupled with the relatively large and rigid delivery systems of the previous generation FD, can challenge the proper placement of the device, which may require the use of multiple devices for adequate neck coverage, increasing the risk of ischemic complications. ${ }^{56}$ Previous authors have recommended to slightly oversize FD selection for highly tortuous vessels to account for device foreshortening ${ }^{44}$; however, this can lead to lengthening of the FD, decreasing its metal coverage and increasing porosity, thus decreasing its effectiveness. This was observed in a meta-analysis by Cagnazzo et al for distal aneurysms using previous generations of FDs, in which a higher occlusion trend was observed with the use of multiple FD than single FD in 484 treated aneurysms (OR 2.3, 95\% CI 0.8 to 7.2; $\mathrm{p}=0.08),{ }^{55}$ likely associated with device oversizing.

Newer generation intracranial FDs like FRED Jr., p48MW and SVB have been introduced to address the technical difficulties with previous generations of FD in distal vessels. Their lower profile and deliverability facilitate their navigation through the small tortuous vessels to treat aneurysms in challenging distal locations (figure 2). Their more stable behavior allows a single FD to achieve occlusion rates similar to those seen with multiple
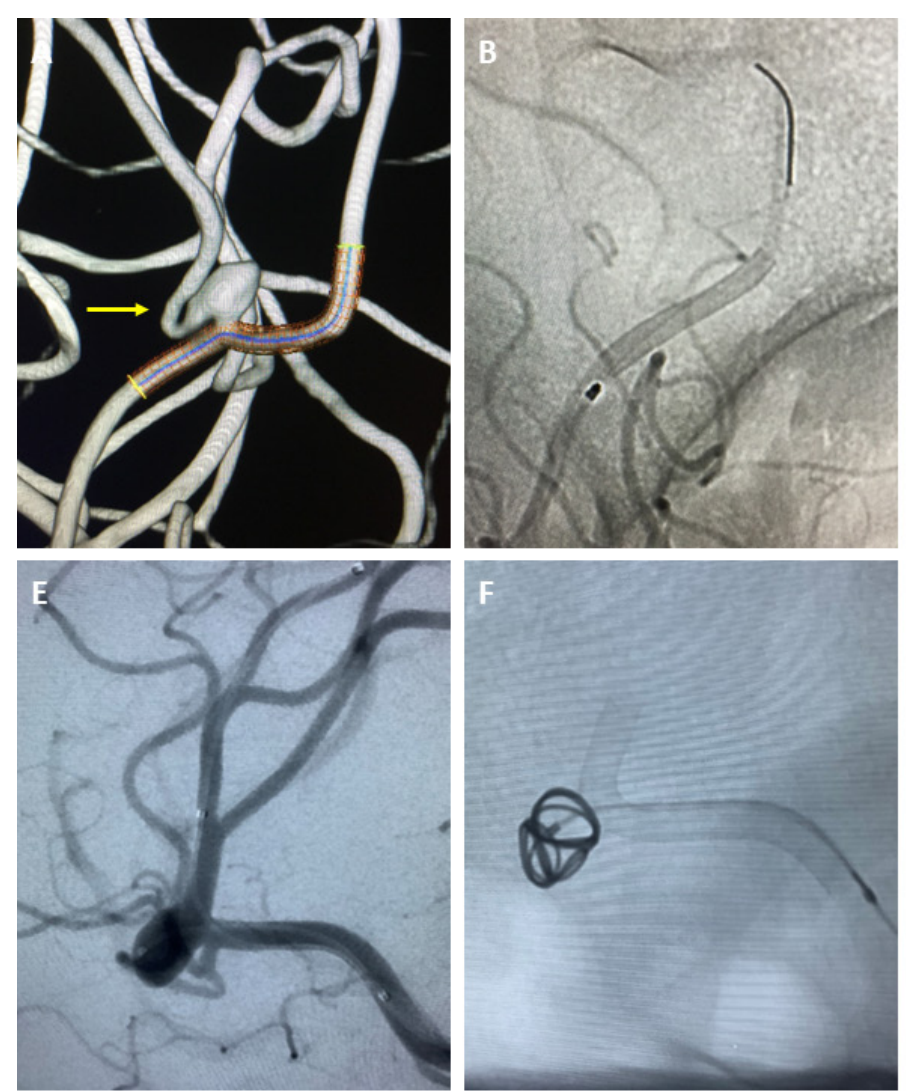
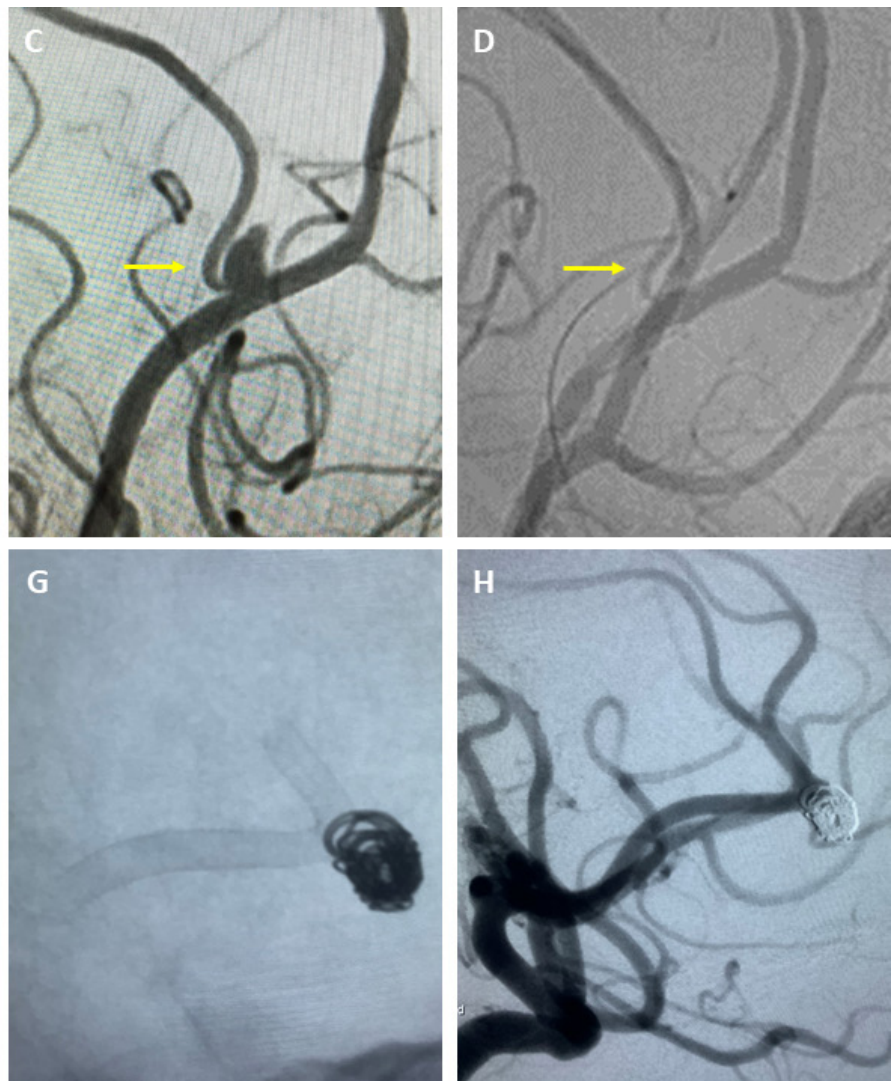

Figure 2 Two cases of embolization of pericallosal aneurysms with Silk Vista Baby (SVB), the second one with coadjutant coiling. (A) 3D reconstruction showing a pericallosal aneurysm with a branch arising from the aneurysm neck (arrow) and pre-treatment device selection of adequate FD size. (B) Complete delivery of a $2.5 \times 15 \mathrm{~mm}$ SVB with adequate wall apposition using the Gama-17 microcatheter. (C) Post-procedure DSA angiogram demonstrating adequate wall apposition with patency of the neck branch (arrow). (D) Complete obliteration of pericallosal aneurysm with patency of the parent vessel and branch (arrow) at 6 months follow-up on DSA. (E) DSA showing another pericallosal aneurysm, access with a Gama-17 microcatheter placed distal to the aneurysm and Echelon-10 microcatheter placed in the aneurysm. (F) Native fluoroscopy demonstrating introduction of the first coil and complete deployment of a $2.5 \times 20 \mathrm{~mm}$ SVB jailing the Echelon-10. (G) Post-procedure native fluoroscopy showing adequate SVB implantation and coiling. (H) Final angiography with complete aneurysm occlusion and patency of the parent vessels and branches. DSA, digital subtraction angiography; FD, flow diverter. 

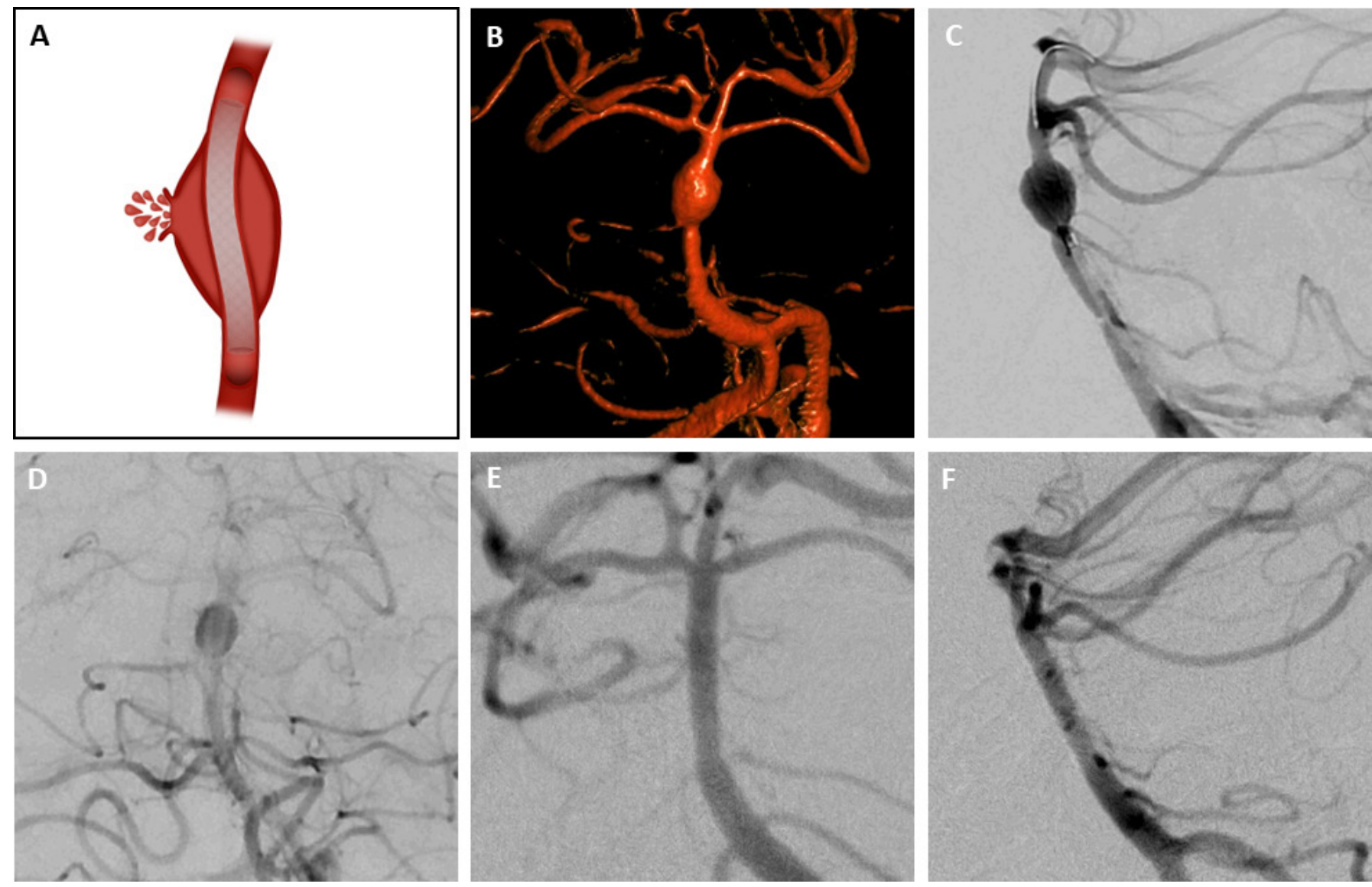

Figure 3 Ruptured basilar fusiform aneurysm treated with Pipeline Embolization Device (PED). (A) Illustration of FD in ruptured fusiform aneurysm. (B) 3D AP view of basilar aneurysm with a side wall perforator. (C) Lateral view of PED deployment at the distal portion of the basilar artery. (D) DSA AP view post-procedure angiography showing patent basilar flow and stagnation of the aneurysm with patency of parent vessel and side wall branch. (E, F) AP and lateral angiographic follow-up at 6 months with complete obliteration of the basilar aneurysm with remodeling of the parent vessel. AP, anteroposterior; DSA, digital subtraction angiography; FD, flow diverter.

FDs in previous generations. ${ }^{38434447}$ Furthermore, their lower profile may also open a window for a new therapeutic approach using FD assisted coiling embolization to achieve immediate aneurysm protection and a permanent obliteration of distal aneurysms (figure 2). ${ }^{43}$ This concept, although promising, requires further careful investigation.

\section{Flow diverters in acutely ruptured intracranial aneurysms}

FDs have been introduced as an alternative to endovascular coiling and surgical clipping in the treatment of selected acutely ruptured complex IA (figure 3). The thin and fragile walls of blister aneurysms, the lack of a true neck in dissecting and fusiform aneurysms, and the coil mass instability in wide-neck and bifurcation aneurysms make these lesions challenging to treat with conventional clipping and endovascular coiling. ${ }^{57}$ However, the use of FDs in acute subarachnoid hemorrhage (aSAH) remains controversial, as DAPT therapy is needed to prevent ischemic complications with FDs and the dome obliteration might not occur immediately, increasing the risk of hemorrhagic complications. In a recent meta-analysis, 223 patients with acutely ruptured IA treated with FDs were evaluated. Results showed an immediate angiographic occlusion rate of $32 \%$ and a long-term adequate aneurysm occlusion rate of $88.9 \%$ at a mean radiologic follow-up of 9.6 months. Overall, ischemic/thromboembolic and hemorrhagic complications were $8 \%$ and $7 \%$, respectively, being more common with saccular aneurysms. Complications overall were associated with posterior circulation aneurysms and treatment with multiple stents. ${ }^{57}$

Newer specialized devices with antithrombotic coating such as the Pipeline Shield, p48_HPC and the Derivo have been introduced as potential alternatives to be used with SAPT, although there is no randomized trial on the topic yet. ${ }^{58} 59$ In a series of 14 patients with acutely ruptured IA treated with Pipeline Shield and SAPT, aneurysmal rebleeding occurred in two patients (14.3\%) with one of them dying. The highest number of hemorrhagic (14.3\%) and ischemic events (14.3\%) were seen in the group treated with daily aspirin plus postoperative heparin infusion, whereas one ischemic event $(7.1 \%)$ was observed with the use of daily aspirin only. No complications were observed with the use of aspirin twice daily. The complete/near complete occlusion rate following 7 days after aSAH was $85.7 \% .^{58}$ For the p48_HPC, eight patients with aSAH were treated with SAPT. Four patients $(50 \%)$ developed intraprocedural thrombus formation, and two patients $(25 \%)$ died of refractory cerebral vasospasm during the postoperative period. No rebleeding from the treated aneurysm occurred. At a mean follow-up of 6 months, five out of six patients (83\%) showed complete aneurysm occlusion. ${ }^{59}$ Although no studies with SAPT have been done yet for the Derivo, 10 patients with aSAH were treated with Derivo and DAPT and showed a complete aneurysm occlusion of $90 \%$ at a median 223 days follow-up. One (10\%) thrombotic event was observed in the post-procedural period in a clopidogrel hypo-responder. No mortality was reported. ${ }^{50}$ 


\section{Flow diverters in posterior circulation aneurysms}

The use of FDs in the posterior circulation has been associated with higher morbidity and mortality. ${ }^{25} 6061$ This may be partially explained by the high amount of perforator vessels originating in this area that could potentially become occluded, leading to critical infarcts. Additionally, the large proportion of thrombosed non-saccular aneurysms arising in this location pose a risk of perforator or stent occlusion due to thrombus migration.

In a single center experience of 12 patients with vertebrobasilar fusiform aneurysms treated with PED, 10 (83\%) patients showed a complete aneurysm occlusion at a mean follow-up of 14.5 months. One (8.3\%) postprocedural complication was reported, likely due to vertebral artery perforator occlusion. The authors attributed their lower ischemic complication rate to the exclusion of holobasilar aneurysms from the study, the strict PFT, the limited number of FD used (mean 1.67 devices), and the use of coadjutant coiling performed in six cases. ${ }^{62}$ Furthermore, Bender et al showed in 55 patients with posterior circulation aneurysms treated with PED a complete occlusion rate of $68 \%$ and $78 \%$ at 6 months and 12 months, respectively. Five (9\%) major strokes and two (4\%) mortalities were reported. The authors discussed the use of DAPT for life in patients undergoing posterior circulation FD, as well as systemic heparinization for 24 hours post-embolization to decrease the risk of long-term ischemic complications. ${ }^{60}$ Importantly, Taschner et al suggested that the clinical course of posterior fossa aneurysm is highly dependent on the time the treatment with FD is proposed. Early treatment in mild or absent symptoms in large and fusiform aneurysms of the posterior fossa can improve clinical results at medium and long term, whereas in highly symptomatic patients no treatment, including FD, has been proved to improve the fatal evolution of these patients. In their multicenter study of 53 patients undergoing SS embolization, a premorbid modified Rankin Scale of 3-5 was associated with mortality (HR 17.11, 95\% CI 2.69 to $109.02 ; \mathrm{p}=0.003)$. The complete occlusion rate was $66 \%$ at a mean 11.3 months follow-up. Seven procedural complications (13\%) and seven deaths (13\%) were reported. ${ }^{61}$

We believe more studies are needed in the controversial and still unsolved topic of FD in vertebrobasilar fusiform (figure 3) and giant aneurysms.

\section{Flow diverters in carotid cavernous fistulas}

Use of FD in the treatment of direct carotid cavernous fistulas (CCFs) along with adjuvant trans-arterial or trans-venous embolization is based on the concept of reconstruction of the defect in the arterial wall which led to the fistula formation (figure 4). Wendl et al described treatment of direct carotid cavernous fistulas primarily using FD with additional trans-arterial or trans-venous coil embolization performed in 14 patients. A total of 59 FDs were deployed (mean 4.12 FD per patient) and included 24 PED and 35 p64 devices. Three patients demonstrated immediate occlusion of the CCF, four patients had significant reduction in their shunting, and half the patients demonstrated a minor leak. At median 20 months follow-up, six out of the 14 patients demonstrated complete occlusion of the CCF and the rest needed re-treatment. ${ }^{63}$ Baranoski et al described their experience of using Pipeline devices along with trans-arterial or trans-venous embolization in the treatment of direct carotid cavernous fistulas in five patients, with a $100 \%$ success rate. A mean of 1.4 devices were used per patient in their study. ${ }^{64}$
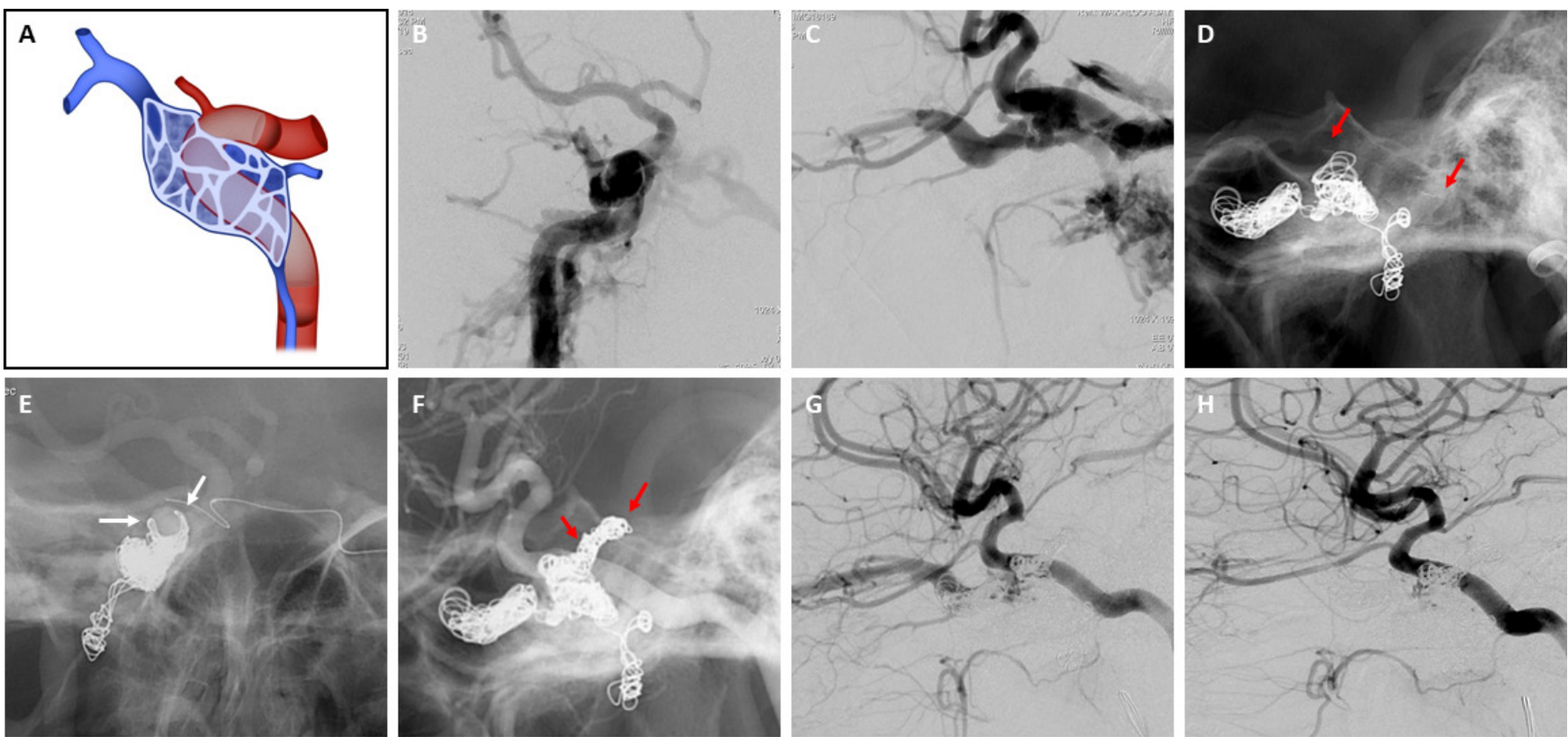

Figure 4 Embolization of a traumatic carotid-cavernous fistula with the Surpass Streamline (SS) Embolization Device. (A) Illustration of FD in the ICA for the treatment of CCF. (B, C) Pre-embolization DSA magnified views of CCF demonstrating shunting from the ICA cavernous segment into the superior ophthalmic vein, intercavernous sinus and pterygoid plexus. (D) Fluoroscopy demonstrating coil mass within the cavernous sinus and superior ophthalmic vein plus two telescoping SS (red arrows). (E) Oblique fluoroscopic view demonstrating the SS (white arrows) protecting the cavernous carotid segment with the coil mass around the carotid fistula point and access of Echelon microcatheter using transvenous approach. (F) Fluoroscopy of lateral view demonstrating additional coils (red arrows) with patency of the ICA. (G) Post-procedure DSA lateral view with minimal shunting of the superior ophthalmic vein. $(\mathrm{H})$ Third day follow-up angiogram lateral view with complete occlusion of the CCF and patency of the parent vessel. CCF, carotid cavernous fistula; DSA, digital subtraction angiography; FD, flow diverter; ICA, internal carotid artery. 


\section{CONCLUSION}

FDs have become the new frontier in endovascular treatment of a large proportion of IA. Their widespread use and experience, user-friendly features and rapid technological evolution have created a unique opportunity to continue expanding their use to cerebrovascular pathology other than that for which they were originally designed. The excitement should be balanced with caution and robust research to demonstrate the safety and efficacy of FDs over traditional endovascular and surgical approaches in off-label scenarios before their use is widely spread. The sheer variety of devices available can be overwhelming and requires neurointerventionalists to be technically proficient in at least one or two of them, while maintaining some knowledge and familiarity of the advantages/limitations of others to use if the clinical scenario so demands it. As the use of FDs in a larger variety of clinical scenarios increases, design customization is needed for specific aneurysm anatomy, location and morphology to cover a broader spectrum of clinical indications.

\section{Author affiliations}

'Department of Neurology, The University of lowa Hospitals and Clinics, lowa City, lowa, USA

${ }^{2}$ Interventional Neuroradiology/Endovascular Neurosurgery, Hospital Clínico Universitario de Valladolid, Valladolid, Spain

${ }^{3}$ Department of Diagnostic and Therapeutic Neuroradiology, Essex Center for Neurological Sciences, Queen's University Hospital, Romford, UK

${ }^{4}$ Department of Interventional Neuroradiology, Hospital Clinic de Barcelona, Barcelona, Catalunya, Spain

${ }^{5}$ Department of Neuroradiology, Instituto Oulton, Córdoba, Argentina

${ }^{6}$ Division of Neuroradiology, Joint Department of Medical Imaging, University of Toronto, Toronto, Ontario, Canada

${ }^{7}$ Division of Neurosurgery, Department of Surgery, University of Toronto, Toronto, Ontario, Canada

${ }^{8}$ Department of Radiology, Hacettepe University Hospitals, Ankara, Turkey

${ }^{9}$ Department of Neurointerventional Radiology, Beth Israel Lahey Health, Tufts University School of Medicine, Burlington, Massachusetts, USA

${ }^{10}$ Department of Radiology and Neurosurgery, The University of lowa Hospitals and Clinics, lowa City, lowa, USA

\section{Twitter Mario Martínez-Galdámez @Doctorgaldamez}

Contributors All authors contributed to the conception, data gathering, and drafting of the manuscript, along with manuscript oversight and administrative support. All authors critically reviewed the manuscript and approved its final submission.

Funding The authors have not declared a specific grant for this research from any funding agency in the public, commercial or not-for-profit sectors.

Disclaimer Mario Martinez-Galdamez is a consultant for Medtronic, Balt Extrusion and Stryker Neurovascular. Juan Macho is a consultant for Stryker Neurovascular. Ajay K Wakhloo is a consultant for Stryker Neurovascular. Vitor M Pereira is a consultant for Balt Extrusion and Stryker Neurovascular. Santiago Ortega-Gutierrez is a consultant for Medtronic and Stryker Neurovascular. Sudeepta Dandapat, Alan Mendez-Ruiz, Gustavo Foa Torres and Anil Arat do not have a financial interest in any of the procedures, drugs or devices described in this article.

\section{Competing interests None declared.}

Patient consent for publication Not required.

Provenance and peer review Commissioned; externally peer reviewed.

ORCID iDs

Sudeepta Dandapat http://orcid.org/0000-0003-3744-7845

Mario Martínez-Galdámez http://orcid.org/0000-0002-8024-4712

Santiago Ortega-Gutierrez http://orcid.org/0000-0003-3744-7845

\section{REFERENCES}

1 Guglielmi G, Viñuela F, Dion J, et al. Electrothrombosis of saccular aneurysms via endovascular approach. Part 2: preliminary clinical experience. J Neurosurg 1991;75:8-14.

2 Molyneux AJ, Kerr RSC, Yu L-M, et al. International subarachnoid aneurysm trial (ISAT) of neurosurgical clipping versus endovascular coiling in 2143 patients with ruptured intracranial aneurysms: a randomised comparison of effects on survival, dependency, seizures, rebleeding, subgroups, and aneurysm occlusion. Lancet 2005;366:809-17.
3 Darsaut TE, Findlay JM, Magro E, et al. Surgical clipping or endovascular coiling for unruptured intracranial aneurysms: a pragmatic randomised trial. J Neurol Neurosurg Psychiatry 2017;88:663-8.

4 Raymond J, Guilbert F, Weill A, et al. Long-term angiographic recurrences after selective endovascular treatment of aneurysms with detachable coils. Stroke 2003:34:1398-403.

5 Cha KS, Balaras E, Lieber BB, et al. Modeling the interaction of coils with the local blood flow after coil embolization of intracranial aneurysms. J Biomech Eng 2007;129:873-9.

6 Wakhloo AK, Lanzino G, Lieber BB, et al. Stents for intracranial aneurysms: the beginning of a new endovascular era? Neurosurgery 1998;43:377-9.

7 Wakhloo AK, Lieber BB. The beginnings of flow diversion: a historical review. In: Park M, Taussky P, Albuquerque FC, et al, eds. Flow diversion of cerebral aneurysms. New York: Thieme, 2018

8 Wakhloo AK, Gounis MJ. Revolution in aneurysm treatment: flow diversion to cure aneurysms: a paradigm shift. Neurosurgery 2014;61:111-20.

9 Sadasivan C, Cesar L, Seong J, et al. An original flow diversion device for the treatment of intracranial aneurysms: evaluation in the rabbit elastase-induced model. Stroke 2009:40:952-8.

10 Lieber BB, Sadasivan C. Endoluminal scaffolds for vascular reconstruction and exclusion of aneurysms from the cerebral circulation. Stroke 2010;41:S21-5.

11 Lieber BB, Stancampiano AP, Wakhloo AK. Alteration of hemodynamics in aneurysm models by stenting: influence of stent porosity. Ann Biomed Eng 1997;25:460-9.

12 Aenis M, Stancampiano AP, Wakhloo AK, et al. Modeling of flow in a straight stented and nonstented side wall aneurysm model. J Biomech Eng 1997;119:206-12.

13 Fernandez H, Macho JM, Blasco J, et al. Computation of the change in length of a braided device when deployed in realistic vessel models. Int I Comput Assist Radiol Surg 2015;10:1659-65.

14 Ospel JM, Gascou G, Costalat V, et al. Comparison of Pipeline Embolization Device sizing based on conventional $2 \mathrm{D}$ measurements and virtual simulation using the Sim\&Size software: an agreement study. AJNR Am J Neuroradiol 2019;40:524-30.

15 Fiorella D, Lylyk P, Szikora I, et al. Curative cerebrovascular reconstruction with the pipeline embolization device: the emergence of definitive endovascular therapy for intracranial aneurysms. J Neurointerv Surg 2009;1:56-65.

16 Marosfoi M, Langan ET, Strittmatter L, et al. In situ tissue engineering: endothelial growth patterns as a function of flow diverter design. J Neurointerv Surg 2017;9:994.

17 Kallmes DF, Ding YH, Dai D, et al. A new endoluminal, flow-disrupting device for treatment of saccular aneurysms. Stroke 2007;38:2346-52.

18 Brinjikji W, Lanzino G, Cloft HJ, et al. Platelet testing is associated with worse clinical outcomes for patients treated with the Pipeline Embolization Device. AJNR Am J Neuroradiol 2015;36::2090.

19 Ajadi E, Kabir S, Cook A, et al. Predictive value of platelet reactivity unit (PRU) value for thrombotic and hemorrhagic events during flow diversion procedures: a metaanalysis. J Neurointerv Surg 2019;11:1123.

20 Adeeb N, Griessenauer CJ, Foreman PM, et al. Use of platelet function testing before Pipeline Embolization Device placement: a multicenter cohort study. Stroke 2017;48:1322-30.

21 Cheung NK, Carr MW, Ray U, et al. Platelet function testing in neurovascular procedures: tool or gimmick? Intervent Neurol 2019;8:123-34.

22 Nelson PK, Lylyk P, Szikora I, et al. The Pipeline Embolization Device for the intracrania treatment of aneurysms trial. AJNR Am J Neuroradiol 2011;32:34-40.

23 Becske T, Brinjikji W, Potts MB, et al. Long-term clinical and angiographic outcomes following Pipeline Embolization Device treatment of complex internal carotid artery aneurysms: five-year results of the Pipeline for Uncoilable or Failed Aneurysms trial. Neurosurgery 2017;80:40-8

24 Becske T, Kallmes DF, Saatci I, et al. Pipeline for uncoilable or failed aneurysms: results from a multicenter clinical trial. Radiology 2013;267:858-68.

25 Kallmes DF, Hanel R, Lopes D, et al. International retrospective study of the Pipeline Embolization Device: a multicenter aneurysm treatment study. AJNR Am J Neuroradiol 2015;36:108-15.

26 Kallmes DF, Brinjikji W, Boccardi E, et al. Aneurysm study of Pipeline in an observational registry (ASPIRe). Interv Neurol 2016;5:89-99.

27 Brasiliense LBC, Aguilar-Salinas P, Lopes DK, et al. Multicenter study of pipeline flex for intracranial aneurysms. Neurosurgery 2019;84:E402-9.

28 Hanel RA, Kallmes DF, Lopes DK, et al. Prospective study on embolization of intracranial aneurysms with the Pipeline device: the PREMIER study 1 year results. J Neurointerv Surg 2020;12:62.

29 Girdhar G, Andersen A, Pangerl E, et al. Thrombogenicity assessment of Pipeline Flex, Pipeline Shield, and FRED flow diverters in an in vitro human blood physiological flow loop model. J Biomed Mater Res A 2018;106:3195-202.

30 Rice H, Martínez Galdámez M, Holtmannspötter M, et al. Periprocedural to 1-year safety and efficacy outcomes with the Pipeline Embolization Device with Shield technology for intracranial aneurysms: a prospective, post-market, multi-center study. J Neurointerv Surg 2020:neurintsurg-2020-015943.

31 Trivelato FP, Wajnberg E, Rezende MTS, et al. Safety and effectiveness of the Pipeline Flex Embolization Device with Shield technology for the treatment of intracranial aneurysms: midterm results from a multicenter study. Neurosurgery 2019;87:104-11 
32 De Vries J, Boogaarts J, Van Norden A, et al. New generation of flow diverter (Surpass) for unruptured intracranial aneurysms: a prospective single-center study in 37 patients. Stroke 2013;44:1567-77.

33 Wakhloo AK, Lylyk P, de Vries J, et al. Surpass flow diverter in the treatment of intracranial aneurysms: a prospective multicenter study. AJNR Am J Neuroradiol 2015;36:98-107.

34 Meyers PM, Coon AL, Kan PT, et al. SCENT trial. Stroke 2019;50:1473-9.

35 Orru E, Rice H, De Villiers L, et al. First clinical experience with the new Surpass Evolve flow diverter: technical and clinical considerations. J Neurointerv Surg 2020 doi:10.1136/neurintsurg-2019-015734. [Epub ahead of print: 12 Feb 2020].

36 Killer-Oberpfalzer M, Kocer N, Griessenauer CJ, et al. European multicenter study for the evaluation of a dual-layer flow-diverting stent for treatment of wide-neck intracranial aneurysms: the European Flow-Redirection Intraluminal Device Study. AJNR Am J Neuroradiol 2018;39:841-7.

37 Pierot L, Spelle L, Berge J, et al. SAFE study (safety and efficacy analysis of FRED embolic device in aneurysm treatment): 1-year clinical and anatomical results. J Neurointerv Surg 2019;11:184.

38 Möhlenbruch MA, Kizilkilic 0, Killer-Oberpfalzer M, et al. Multicenter experience with FRED Jr flow re-direction endoluminal device for intracranial aneurysms in small arteries. AJNR Am J Neuroradiol 2017;38:1959-65.

39 Pumar JM, Banguero A, Cuellar $\mathrm{H}$, et al. Treatment of intracranial aneurysms with the Silk embolization device in a multicenter study. A retrospective data analysis. Neurosurgery 2017;81:595-601.

40 Lubicz B, Collignon L, Raphaeli G, et al. Flow-diverter stent for the endovascular treatment of intracranial aneurysms: a prospective study in 29 patients with 34 aneurysms. Stroke 2010;41:2247-53.

41 Foa Torres G, Roca F, Noguera A, et al. Silk flow-diverter stent for the treatment of complex intracranial aneurysms: a one-year follow-up multicenter study. Interv Neuroradiol 2018;24:357-62.

42 Lubicz B, Van der Elst O, Collignon L, et al. Silk flow-diverter stent for the treatment of intracranial aneurysms: a series of 58 patients with emphasis on long-term results. AJNR Am J Neuroradiol 2015;36:542-6.

43 Martínez-Galdámez M, Biondi A, Kalousek V, et al. Periprocedural safety and technica outcomes of the new Silk Vista Baby flow diverter for the treatment of intracranial aneurysms: results from a multicenter experience. J Neurointerv Surg 2019;11:723-7.

44 Schob S, Hoffmann K-T, Richter C, et al. Flow diversion beyond the circle of Willis: endovascular aneurysm treatment in peripheral cerebral arteries employing a novel low-profile flow diverting stent. J Neurointerv Surg 2019;11:1227.

45 Briganti F, Leone G, Ugga L, et al. Mid-term and long-term follow-up of intracranial aneurysms treated by the p64 flow modulation device: a multicenter experience. J Neurointerv Surg 2017;9:70-6.

46 Morais R, Mine B, Bruyère PJ, et al. Endovascular treatment of intracranial aneurysms with the p64 flow diverter stent: mid-term results in 35 patients with 41 intracranial aneurysms. Neuroradiology 2017;59:263-9.

47 Bhogal P, Bleise C, Chudyk J, et al. The p48MW flow diverter-initial human experience Clin Neuroradio/ 2019. doi:10.1007/s00062-019-00827-8. [Epub ahead of print: 21 Aug 2019].
48 Lenz-Habijan T, Bhogal P, Peters M, et al. Hydrophilic stent coating inhibits platelet adhesion on stent surfaces: initial results in vitro. Cardiovasc Intervent Radiol 2018;41:1779-85.

49 Bhogal P, Bleise C, Chudyk J, et al. The p48_HPC antithrombogenic flow diverter: initial human experience using single antiplatelet therapy. J Int Med Res 2020;48:300060519879580.

50 Goertz L, Dorn F, Kraus B, et al. Safety and efficacy of the Derivo embolization device for the treatment of ruptured intracranial aneurysms. J Neurointerv Surg 2019;11:290

51 Trivelato FP, Abud DG, Ulhôa AC, et al. Derivo embolization device for the treatment of intracranial aneurysms. Stroke 2019;50:2351-8.

52 Zhou Y, Yang P-F, Fang Y-B, et al. A novel flow-diverting device (Tubridge) for the treatment of 28 large or giant intracranial aneurysms: a single-center experience. AJNR Am J Neuroradiol 2014;35:2326-33.

53 Al Kasab S, Guerrero WR, Nakagawa D, et al. Safety and efficacy of the Pipeline Embolization Device use in the outside circle of Willis located intracranial aneurysms: a single-center experience. Interv Neurol 2020;8:83-91.

54 Primiani CT, Ren Z, Kan P, et al. A2, M2, P2 aneurysms and beyond: results of treatment with Pipeline Embolization Device in 65 patients. J Neurointerv Surg 2019;11:903.

55 Cagnazzo F, Perrini P, Dargazanli C, et al. Treatment of unruptured distal anterior circulation aneurysms with flow-diverter stents: a meta-analysis. AJNR Am J Neuroradiol 2019;40:687-93.

56 Brinjikji W, Lanzino G, Cloft HJ, et al. Risk factors for ischemic complications following Pipeline Embolization Device treatment of intracranial aneurysms: results from the IntrePED study. AJNR Am J Neuroradiol 2016;37:1673.

57 Cagnazzo F, di Carlo DT, Cappucci M, et al. Acutely ruptured intracranial aneurysms treated with flow-diverter stents: a systematic review and meta-analysis. AJNR Am J Neuroradiol 2018;39:1669-75.

58 Manning NW, Cheung A, Phillips TJ, et al. Pipeline Shield with single antiplatelet therapy in aneurysmal subarachnoid haemorrhage: multicentre experience. $J$ Neurointerv Surg 2019;11:694-8.

59 Aguilar-Perez M, Hellstern V, AlMatter M, et al. The p48 flow modulation device with hydrophilic polymer coating (HPC) for the treatment of acutely ruptured aneurysms: early clinical experience using single antiplatelet therapy. Cardiovasc Intervent Radiol 2020;43:740-8.

60 Bender MT, Colby GP, Jiang B, et al. Flow diversion of posterior circulation cerebral aneurysms: a single-institution series of 59 cases. Neurosurgery 2019;84:206-16.

61 Taschner CA, Vedantham S, de Vries J, et al. Surpass flow diverter for treatment of posterior circulation aneurysms. AJNR Am J Neuroradiol 2017:38:582-9.

62 Natarajan SK, Lin N, Sonig A, et al. The safety of Pipeline flow diversion in fusiform vertebrobasilar aneurysms: a consecutive case series with longer-term follow-up from a single US center. J Neurosurg 2016;125:111-9.

63 Wendl CM, Henkes H, Martinez Moreno R, et al. Direct carotid cavernous sinus fistulae: vessel reconstruction using flow-diverting implants. Clin Neuroradiol 2017;27:493-501.

64 Baranoski JF, Ducruet A, Przbylowski CJ, et al. Flow diverters as a scaffold for treating direct carotid cavernous fistulas. J Neurointerv Surg 2019;11:1129-34. 